\title{
Optimum Adaptive Piecewise Linearization: An Estimation Approach in Wind Power
}

\author{
Masoud Vaezi, Pardis Khayyer, Member, IEEE, and Afshin Izadian, Senior Member, IEEE
}

\begin{abstract}
This paper introduces an effective piecewise linearization technique to obtain an estimation of nonlinear models when their input-output domains include multidimensional operating points. The algorithm of a forward adaptive approach is introduced to identify the effective operating points for model linearization and adjust their domains for the maximum coverage and the minimum model linearization error. The technique obtains a minimum number of linearized models and the continuity of their domains. The algorithm also yields global minimum model linearization error. The introduced algorithm is formulated for a wind power transfer system for a 2-D set of input domains. The linearization error can be arbitrarily minimized in exchange for a higher number of models. The results demonstrate a significant improvement in the linearization of nonlinear models.
\end{abstract}

Index Terms-Adaptive continuity, nonlinear hydraulic wind power, piecewise linearization.

\section{INTRODUCTION}

$\mathbf{L}$ INEARIZATION of nonlinear systems allows the use of established linear system analysis and control techniques such as Laplace transform, Fourier transform, and superposition principles [1]. The general approach in linearization of a nonlinear system can be categorized into two major groups, exact linearization and approximate linearization. Among others, exact linearization [2] can be obtained using inputstate feedback linearization, input-output feedback linearization [3], and state space linearization [4]. The approach is to algebraically transform nonlinear system dynamics into (fully or partly) linear models. The zero dynamics of these systems must be stable. The main drawbacks are the need for a fairly accurate system model, as well as the need to solve potentially difficult nonlinear partial differential equations.

On the other hand, approximate linearization methods such as Jacobian linearization, optimal linearization [5], and fuzzy linearization [6] can estimate nonlinear models in a certain region within a given tolerance [7]. To obtain a linear approximation of a nonlinear model with a wide range of operating points, the approximate linearization methods can be employed

Manuscript received November 17, 2014; revised August 29, 2015 and January 13, 2016; accepted May 19, 2016. Manuscript received in final form May 31, 2016. Recommended by Associate Editor T. Parisini.

M. Vaezi and A. Izadian are with the Energy Systems and Power Electronics Lab, Purdue School of Engineering and Technology, Indianapolis, IN 46202 USA (e-mail: mvaezi@iupui.edu; aizadian@iupui.edu).

P. Khayyer was with the Department of Electrical and Computer Engineering, The Ohio State University, Columbus, OH 43210 USA (e-mail: khayyer.1@osu.edu).

Color versions of one or more of the figures in this paper are available online at http://ieeexplore.ieee.org.

Digital Object Identifier 10.1109/TCST.2016.2575780 locally to perform a piecewise linearization. This approach captures nonlinearities around equilibrium points. In addition, research on linearization of stochastic dynamic systems under excitations has led to progress in statistical and equivalent linearization. The objective of statistical linearization is to replace the nonlinear elements in a model by linear forms where the coefficients of linearization can be generated according to a specified criterion of linearization in an iterative procedure. In equivalent linearization, the direct minimization of a criterion based on the output probability density functions of a dynamic nonlinear system is proposed [8].

Various methods of piecewise linearization have been introduced in the literature and most of them have been effectively utilized in engineering areas such as digital and analog circuits [9], [10] and computer programming problems [11], [12]. Approximation of strongly nonlinear systems around multiple operating points using Taylor expansion also leads to the piecewise linearization. The resulting linear models have a lower complexity, fit into well-established tools for linear systems, and are capable of representing arbitrary nonlinear mappings [13]. Once the piecewise linearization is carried out, the set of selected linear models can be utilized in different control areas such as gain scheduling, multiple adaptive control and system analysis, etc. Therefore, the accuracy and quality of piecewise linearization can greatly affect the further system development.

The satisfactory performance of piecewise linearization algorithms depends on: 1) the number of linear models required to represent a nonlinear model and 2) the location of the operating points to obtain the maximum coverage and continuity.

Some methods [14] suggest equal partition of the operating points and selection of fixed number of models that has generated suboptimal results. The extent of these intervals depends on the nonlinearity of the original model. A simple and common linearization strategy consists of building a linear interpolation between samples of the nonlinear function over a uniform partition of its domain. A tradeoff between increasing the approximation accuracy and simplifying the approximation by the minimum number of linearized sectors can be obtained using genetic algorithms [15].

This paper introduces a novel adaptive piecewise linearization algorithm to optimize the location of operating points, minimize the number of piecewise linear models, and preserve the continuity of the nonlinear-representing system. The number of piecewise linear models is determined such that the maximum number of operating points are covered 
with a globally minimized modeling error. A complementary adaptive method is used to maintain the continuity of the linearized models on the overlapped linear model domains. The algorithm is formulated for an example of a nonlinear wind power system with a 2-D input domain.

This paper is organized as follows. Section II introduces adaptive optimum piecewise linearization as a novel algorithm to select the proper operating points and model linearization. A nonlinear model of wind power systems is discussed in Section III. Mathematical modeling of the hydraulic wind power system is represented in Section IV. Section V introduces the 2-D linearization of the wind power transmission system. Finally, Section VI represents the simulations and results.

\section{Adaptive Optimum Piecewise Linearization}

A wide operating range of hydraulic wind power requires several linearized models. An effective model selection is required to cover all the operating points of the system with the best matching on the overlapped areas. An algorithm is introduced to locate the operating points of the system and determine the number of piecewise linearized models. An adaptive technique is used to linearly combine these piecewise linearized models for model selection on overlapped and uncovered areas. The optimization algorithm is as follows.

\section{A. Model Selection}

Step 1: In the first step, the entire range of operating conditions can be divided into individual nodes with proper dimensions that are determined according to the number of inputs. The inputs to the system (disturbance input or controlled input) will specify the operating point of a system. Therefore, a set of values for inputs can represent an operating point, i.e., a set of values for system states. For instance, the whole operating range of a two-input system can be represented in a 2-D surface, which can be discretized to 2-D nodes. Hence, the dimension of nodes can be obtained as $N=\#_{\text {input }}$, where \# shows the number of input variables. The nodes of the domain are considered as operating points.

Step 2: Each operating point (node) potentially forms a linearized model. The obtained linearized model can be used for other neighboring nodes if the linearization error is less than a desired threshold. The domain of each model is determined by examining the operating points and obtaining the error between the original model and that of the linearized model. For instance, Fig. 1 shows the schematic domain of two linearized models around nodes $i_{1 \times N}$ and $j_{1 \times N}$ as $D_{i}$ or $D_{j}$. Domain is defined as a set of neighboring nodes at which the error between the linearized model and the nonlinear model remains less than a threshold $\varepsilon$. The domain of a general node $x_{1 \times N}, D_{x}$, can be expressed as a set of nodes that show a relative low model linearization error as follows:

$$
D_{x}=\left\{\forall x_{1 \times N}|| f(x)-\bar{f}(x) \mid<\varepsilon\right\}
$$

where $f(x)$ is the nonlinear function and $\bar{f}(x)$ is the piecewise linearized function around node $x$. The domain discretization may be considered as an even distribution. However, this

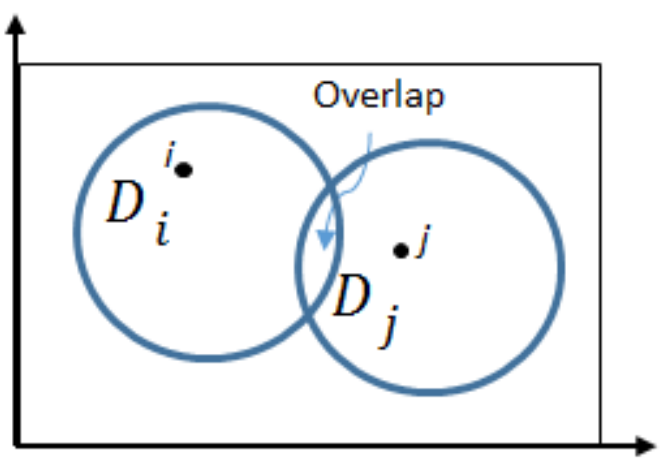

Fig. 1. Overlap between two model domains. Shared nodes in the overlapped area can be selected by both the models. A probability will be associated to each model that involves a node to maintain the continuity $D_{i}$ and $D_{j}$ represent the domains of models $i$ and $j$.

depends on the nonlinear behavior of the system. A higher resolution in the domain nodes may reduce the modeling error. However, a higher number of linearized models may be required. Adaptive mixing can be utilized to maintain the continuity of the domains and reach globally minimum modeling error.

Step 3: Each operating point $x$ is weighted as $\omega_{x}$. The weighting depends on the number of models $n$ that include this particular node in their domain $D_{n}$ as follows:

$$
\omega_{x}=\sum_{n \mid x \in D_{n}} n .
$$

A node with a higher weight is less valuable as it can be expressed with more possible piecewise linearized models. The modified weight for the node $x, \Gamma_{x}$, is inversely scaled and magnified to generate proper resolution for distinct recognition of models as follows:

$$
\Gamma_{x}=\alpha \frac{1}{\omega_{x}^{\beta}}
$$

wherein the positive parameters $\alpha$ and $\beta$ are the gain and shape variables tuned to create the weighting resolution.

Step 4: Model weighting. Accumulative modified weight is obtained according to the summation of the node modified weights $\Gamma_{x}$ existing in the domain of a model, as follows:

$$
\Gamma_{\bar{f}_{i}}=\sum_{x \in D_{x}} \Gamma_{x}, \quad i=1,2, \ldots n
$$

where $\bar{f}_{i}$ is the piecewise linear model of domain $i$.

Step 5: The models are ranked according to their accumulative modified weights. The maximum weighted model is selected as one of the piecewise linearized models as follows:

$$
\bar{f}_{i}=\arg \max \left\{\Gamma_{\bar{f}_{i}}\right\}, \quad i=1,2, \ldots, n .
$$

Step 6: When a model is selected, the nodes associated with the model are nullified (weighted zero) and the models will be weighted and ranked

$$
\left\{\forall x \in D_{x}, \Gamma_{x}=0\right\} .
$$

Step 7: Domain index criterion. Model selection stops if the number of added operating points per added linearized 


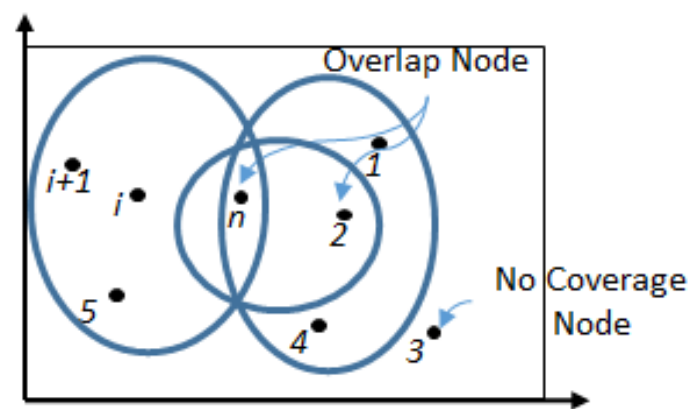

Fig. 2. Node might belong to the domain of a single model, multiple model, and no model of piecewise linearized models.

model is less than the domain index $\delta$. This index indicates the desired collective number of domain nodes in the model selection procedure. A small index stops the consideration of a new model in the linearization process. This means that adding one more model does not include a significant number of nodes to the domain of linearized models. The process stops and since the models were ranked according to the importance of nodes they would involve, stopping the increment of one more linearized model does not introduce a significant modeling linearization error. In addition, adaptive model mixing covers all the operating points. The model selection's exit condition is expressed as follows:

$$
\text { (if } \left.\frac{D_{i+1}}{\sum_{n=1,2, \ldots,(i+1)} D_{n}}<\delta \Rightarrow \bar{f}_{i+1}=\varnothing\right) .
$$

A tradeoff exists between the number of linearized models and the linearization error. In a high-performance linearization in which the deviation of the linearized model from the nonlinear model is limited, a small modeling index $\delta$ should be considered that can potentially lead to a larger number of linearized models.

Depending upon the criterion determined in step 6, there might be nodes that do not belong to any of the piecewise linearized models' domains. There is also the possibility that some nodes belong to more than one piecewise linearized model. To allocate nodes to each model or a combination of models, an adaptive model mixing algorithm is utilized. This algorithm generates a linear combination of models with proper weights that are estimated through an adaptive process that assigns all the operating points (node) to a proper model.

\section{B. Adaptive Linear Continuity}

Optimum model selection, as explained in step 7, may stop prior to covering all the operating points. There might exist overlapping or uncovered nodes by automatically generated piecewise linearized models. Fig. 2 shows $n$ models with selected domains that may have overlaps. There exist nodes that do not belong to any system domain, in which the continuity of domain can be lost.

To mitigate this problem, the nodes in either overlapped or uncovered domains must be allocated to an estimated model that can be generated through an adaptive linear combination

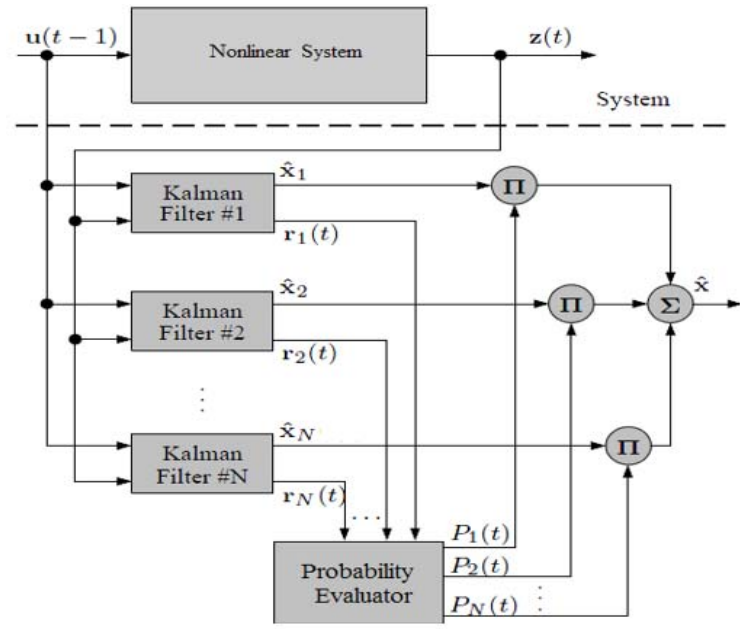

Fig. 3. Adaptive estimation as model-mixing procedure. Each node will have multiple models with designated probabilities. The sum of all model probabilities associated to a node at any time instance is 1 .

of existing models. Adaptive model estimation techniques such as Newton, least square, or multiple model selection have been developed for this purpose [16], [17]. As the nodes may contain uncertainty, noise, or disturbances, Kalman-based models can be used. Adaptive model-based estimation is used to combine the information contained in the local models into a global description of the nonlinear dynamics, and then carry out state estimation by tracking transition online. This method has also been used for fault diagnosis in different applications [18].

Kalman filters are used to estimate the states of linear systems. However, if inaccurate model parameters are used to construct the filter, the state estimate accuracy will degrade and may even diverge [19]. Kalman filter of a linearized model $k$ can be represented [20], [21] as follows:

$$
\begin{aligned}
& \hat{x}_{k}\left(t_{i}^{-}\right)=\Phi_{k} \hat{x}_{k}\left(t_{i-1}^{+}\right)+B_{k} u\left(t_{i-1}\right) \\
& \hat{z}_{k}\left(t_{i}^{-}\right)=H_{k} \hat{x}_{k}\left(t_{i}^{-}\right)
\end{aligned}
$$

where $\hat{x}_{k}$ is the state estimation vector, $\hat{z}_{k}\left(t_{i}^{-}\right)$is the output estimation at time $t_{i}^{-}$of the $i$ th time sample, and $t_{i-1}^{+}$is the time after the measurement update at the $(i-1)$ th time sample. $\Phi_{k}$ is the state transition matrix, $B_{k}$ is the control input matrix, $u$ is the system input vector, $z_{k}$ is the measurement noise, $H_{k}$ is the output matrix, and the state estimation covariance matrix propagation is as follows [22]:

$$
\hat{x}_{k}\left(t_{i}^{+}\right)=\hat{x}_{k}\left(t_{i}^{-}\right)+K_{k}\left(t_{i}\right)\left(z_{T}\left(t_{i}\right)-H_{k} \hat{x}_{k}\left(t_{i}^{-}\right)\right)
$$

where the Kalman gain $K_{k}$ and the covariance matrix $P_{k}$ are updated through the recursive equations, considering the measurement noise covariance $R_{k}$, as follows:

$$
\begin{aligned}
K_{k}\left(t_{i}\right) & =P_{k}\left(t_{i}^{-}\right) H_{k}^{T}\left(H_{k} P_{k}\left(t_{i}^{-}\right) H_{k}^{T}+R_{k}\right)^{-1} \\
P_{k}\left(t_{i}^{+}\right) & =P_{k}\left(t_{i}^{-}\right)-K_{k}\left(t_{i}\right) H_{k} P_{k}\left(t_{i}^{-}\right) .
\end{aligned}
$$

A block diagram of the adaptive model-based estimation scheme is shown in Fig. 3. The input and output data of a plant with a wide range of operating points is collected and 
passed to a bank of Kalman filters. The Kalman bank contains parallel filters constructed to represent a particular model in operating point domains.

The output of each filter is compared with that of the actual plant and the model with the lowest residual represents a close model. The adaptive model-based estimation generates a weighted average of all Kalman estimated values, where the weights are obtained by a posteriori probability of the residual signals considering a history of the input-output variations [23]. The weights are conditional probability values that are generated for each model [20] as follows:

$$
p_{k}\left(t_{i}\right)=\frac{f_{z\left(t_{i}\right) \mid h, z\left(t_{i-1}\right)}\left(z_{i} \mid h_{k}, Z_{i-1}\right) \cdot p_{k}\left(t_{i-1}\right)}{\sum_{j=1}^{K} f_{z\left(t_{i}\right) \mid h, z\left(t_{i-1}\right)}\left(z_{i} \mid h_{k}, Z_{i-1}\right) \cdot p_{j}\left(t_{i-1}\right)}
$$

where

$$
\begin{aligned}
& f_{z\left(t_{i}\right) \mid h, z\left(t_{i-1}\right)}\left(z_{i} \mid h_{k}, Z_{i-1}\right) \\
& \quad=\frac{1}{(2 \pi)^{m / 2}\left|A_{k}\left(t_{i}\right)\right|^{1 / 2}} \exp \left(-\frac{1}{2} r_{k}\left(t_{i}\right)^{T} A_{k}^{-1}\left(t_{i}\right) r_{k}\left(t_{i}\right)\right) .
\end{aligned}
$$

In these equations, $f_{z\left(t_{i}\right) \mid h, z\left(t_{i-1}\right)}\left(z_{i} \mid h_{k}, Z_{i-1}\right)$ is the probability density function of the current measurement $Z\left(t_{i}\right)$ conditioned on the hypothesized status and measurement history $Z\left(t_{i-1}\right)$, based on residual signal $r_{k}$ and $A_{k}$. Residual signal $r_{k}$ is obtained as a difference of the actual plant with that of the linearized model. The output of this block is a vector of probabilities that can be used to weight the state estimates. The output of the algorithm is a probability weighted state estimate that determines the models and their associated weights to be linearly combined.

The adaptive model mixing approach maintains model continuity because the sum of adaptive weights for $n$ linear combination of piecewise models is 1, i.e., $\sum_{i=1, \ldots, n} p_{i}=1$.

Kalman filters are updated in an offline process to increase the convergence rate and reduce model switching.

It can be concluded that the adaptive piecewise linearization of a nonlinear system is a minimum-error linearization process. To analyze this conclusion, assume $\bar{f}_{i}$ represents the linearized model value for segment $i,(i=1, \ldots n)$ of nonlinear system $f(x)$. Adaptive mixing of piecewise linearized systems is represented in the form of $\sum_{i=1, \ldots, n} P_{i} \bar{f}_{i}$, which satisfies $\forall i, 0 \leq P_{i} \leq 1$ and $\sum_{i=1, \ldots, n} p_{i}=1$. These conditions make adaptive piecewise linearized systems a convex model.

The ranked piecewise linearized models $i,(i=1, \ldots n)$ have noninfinite domains $D_{i}<\infty$, where the domain $D_{i}$ is obtained by limiting the linearization error in step 2 . Therefore, adaptive piecewise linearization of a nonlinear system reaches the minimum modeling error over all piecewise models.

\section{FORMULATION FOR NONLINEAR HYDRAULIC WIND POWER TRANSMISSION SYSTEM}

\section{A. System Configuration}

Hydraulic transmission of wind power provides the capability of collecting energy from several wind turbines into one generation unit. In this technique, kinetic energy of the turbine is converted into hydraulic pressurized fluid at the pump [24]. This energy is transferred to the location of the generator and

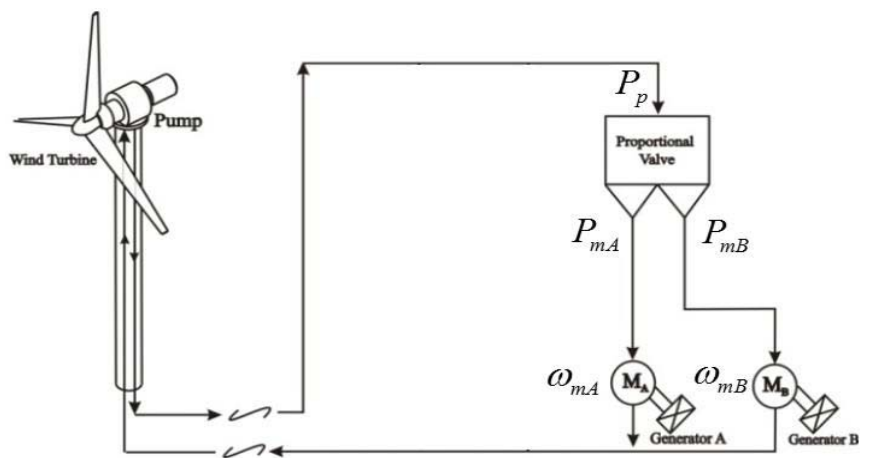

Fig. 4. Schematic of a single wind turbine system. The pump pressure and the outlet pressure at the proportional valve indicate the power distribution between the generators A and B. More pressure is generated because of the torque at the shaft of the generator.

is released to the generator through a hydraulic motor. Fluid control devices such as proportional valves and check valves are required to maintain the desired operating conditions [25]. The system experiences a wide range of wind speeds and accordingly a wide range of valve operations that together cover a large area of possible operating points. At a low wind speed, the system becomes highly nonlinear. The existence of the proportional valve adds to the nonlinear operation of the system by adding three state variables as pressures at the inlet and at the two outlets.

The proportional valve-controlled hydraulic wind power system is a nonlinear dynamic with a minimum of five state variables. A detailed nonlinear model of the hydraulic wind power system is provided in [26] and [27]. The system's input variables cover a wide area of operation. Hence, a high performance linearization technique should consider many operating points and consequently several linearized models to demonstrate the system operation.

The hydraulic transmission system (HTS) comprises of hydraulic pumps, hydraulic motors, transmission lines, and pressure and flow control valves. Hydraulic pumps coupled with the turbines supply flow to the hydraulic circuit. The flow is transferred to ground level and is distributed through a proportional valve between two hydraulic motors. These motors are connected to two generators to generate the main source of power at $60 \mathrm{~Hz}$ (at the main motor) and provide storage capability for excess power (at the auxiliary motor) [28]. Fig. 4 shows a schematic of the introduced HTS.

As the turbine turns, the hydraulic pump pressurizes the fluid medium and flows the liquid in the hydraulic circuitry. The pressurized liquid is split between two motors and the right amount of flow reaches the main hydraulic motor and turns the motor and the generator coupled with it. The circuit is complete as the return line circulates the fluid back to the pump.

\section{B. System Operation}

As the wind speed varies with time, the wind turbine will rotate at a variable speed and generates various amounts of torque on the shaft. This causes a pressure or flow change in the system. On the other hand, the proportional valve is actively controlled to maintain a regulated flow to main 


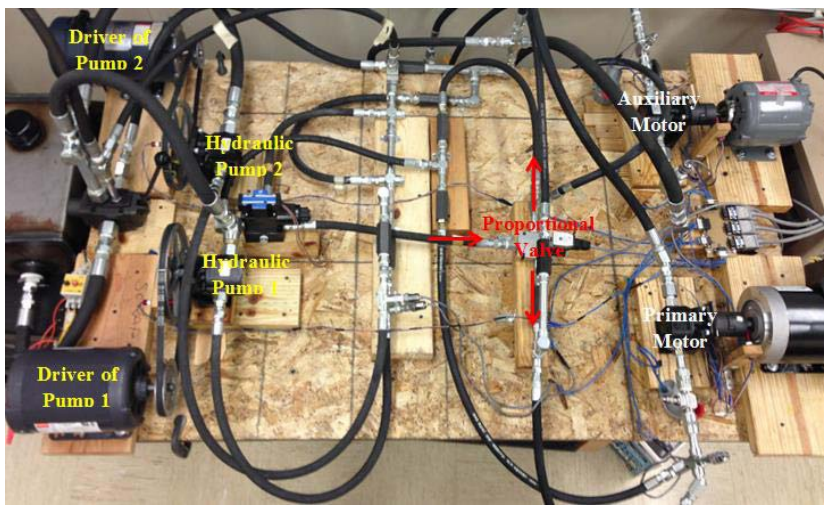

Fig. 5. Experimental setup of the hydrostatic wind power transfer system. Energy Systems and Power Electronics Laboratory, Purdue School of Engineering and Technology. The setup shows two hydraulic pumps that can send the harvested energy of wind through the pressurized medium to hydraulic motors. The proportional valve distributes the flow between the motors to adjust their speed. The hydraulic motors are connected to electric generators. Left: prime mover stage. Middle: distribution. Right: generation.

TABLE I

LIST OF EXPERIMENTAL PROTOTYPE PARAMETERS

\begin{tabular}{|c|c|c|c|}
\hline Symbol & QUANTITY & Value & Unit \\
\hline$D_{p}$ & Pump displacement & 0.722 & $\mathrm{in}^{3} / \mathrm{rev}$ \\
\hline$D_{m A}$ & Primary motor displacement & 0.241 & $\mathrm{in}^{3} / \mathrm{rev}$ \\
\hline$D_{m B}$ & Auxiliary motor displacement & 0.515 & $\mathrm{in}^{3} / \mathrm{rev}$ \\
\hline$I_{m A}$ & Primary motor inertia & 9.6 & lb.in ${ }^{2}$ \\
\hline$I_{m B}$ & Auxiliary motor inertia & 4.8 & lb.in ${ }^{2}$ \\
\hline$B_{m A}$ & Primary motor damping & 0.0141 & lb.in/(rev/min) \\
\hline$B_{m B}$ & Auxiliary motor damping & 0.0115 & lb.in/(rev/min) \\
\hline$K_{p}$ & Pump leakage coefficient & 0.046 & $\mathrm{in}^{3} /($ psi.min $)$ \\
\hline$K_{m A}$ & $\begin{array}{l}\text { Primary motor leakage } \\
\text { coefficient }\end{array}$ & 0.06 & $\mathrm{in}^{3} /(\mathrm{psi} . \mathrm{min})$ \\
\hline$K_{m B}$ & $\begin{array}{l}\text { Auxiliary motor leakage } \\
\text { coefficient }\end{array}$ & 0.01 & in $^{3} /($ psi.min $)$ \\
\hline$v$ & Fluid viscosity & 1.105 & $\mathrm{in}^{2} / \mathrm{s}$ \\
\hline$\beta$ & Fluid bulk modulus & 183695 & psi \\
\hline$\rho$ & Fluid density & 0.0305 & $\mathrm{lbm} / \mathrm{in}^{3}$ \\
\hline
\end{tabular}

the motor under a constant load torque. As the load torque changes, the proportional valve will be adjusted accordingly [29], [30]. Consequently, the hydraulic wind power transmission has the shaft speed and valve position as input variables. Together, the system response and the two inputs as wind speed and valve position cover a large area of operation. Fig. 5 shows the experimental setup as a test-bed in our laboratory. In addition, Table I lists the actual parameters of the experimental setup, which are further used to derive the actual linear state space models.

\section{Nonlinear Mathematical Model}

The state space representation of the hydraulic wind power system can be derived by considering the integrated configuration of the hydraulic components such as pumps, hydraulic motors, proportional valves, and check valves. The nonlinear model of hydraulic circuit components and the nonlinear state space representation of the hydraulic wind energy transfer are also introduced in [26].

Considering the dynamics of each hydraulic component, the governing equations of flow and torque are presented in this section. These equations are used to represent the HTS in the form of nonlinear state space variables defined in vector $x$ as follows:

$$
x=\left[\begin{array}{lllll}
P_{p} & P_{m A} & P_{m B} & \omega_{m A} & \omega_{m B}
\end{array}\right]^{T}
$$

where $P_{p}$ is the pump pressure, $P_{m A}$ is the main motor inlet pressure, $P_{m B}$ is the auxiliary motor inlet pressure, $\omega_{m A}$ is the main motor angular velocity, and $\omega_{m B}$ is the auxiliary motor angular velocity.

The proportional valve consists of one inlet and two outlets. A spool controls the flow passage areas at the outlet orifices. The fluid enters the valve and based on the spool's position, the flow is distributed between the outlets: main and auxiliary. The spool is displaced by electromagnetic force generated from a coil. The governing equation of flow rate for each outlet is obtained from fluid mechanics principles [31], using the pressure as

$$
Q=C_{D} A \sqrt{\frac{2 \Delta P}{\rho}}
$$

where $A$ is the orifice area and $\Delta P$ is the pressure differential across the orifice. $C_{D}$ and $\rho$ are discharge coefficient and the fluid density, respectively. The governing equation for motor flow and delivered toque to the load are

$$
\begin{aligned}
I_{m} \frac{d \omega_{m}}{d t} & =D_{m} P_{m}-C_{v} D_{m} \mu \omega_{m}-T_{l} \\
Q_{m} & =D_{m} \omega_{m}+K_{m s} P_{m} .
\end{aligned}
$$

Considering the input vector $U=\left[\begin{array}{ll}h_{i} & \omega_{p}\end{array}\right]^{T}$, where $\omega_{p}$ is the pump speed and $h_{i}$ is the valve position, the state space model of the system is represented as

$$
\left\{\begin{array}{l}
\dot{x}=f(x)+g(x) u \\
y=h(x)
\end{array}\right.
$$

where functions $f(x)$ and $g(x)$ are expressed as follows:

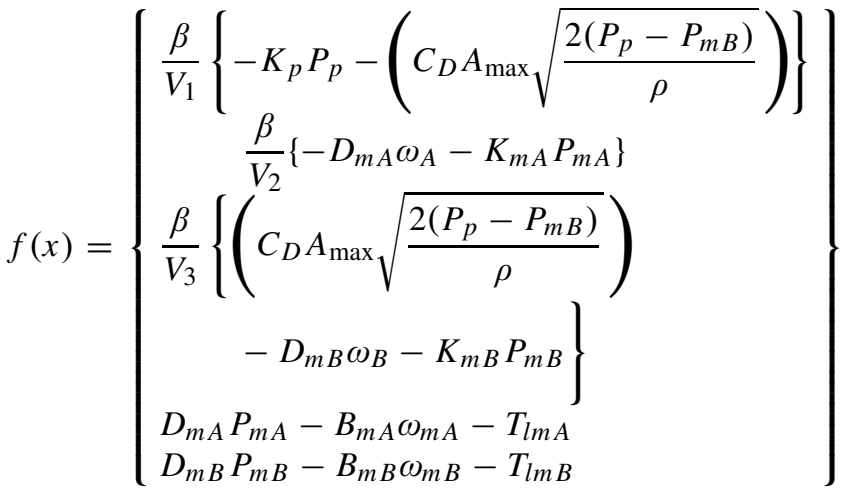




$$
g(x)=\left[\begin{array}{r}
\frac{\beta}{V_{1}}\left\{-\left(C_{D} \frac{A_{\max }}{h_{\max }} \sqrt{\left.\frac{2\left(P_{p}-P_{m A}\right)}{\rho}\right)}\right)\right. \\
\left.+\left(C_{D} \frac{A_{\max }}{h_{\max }} \sqrt{\frac{2\left(P_{p}-P_{m B}\right)}{\rho}}\right)\right\} \\
\frac{\beta}{V_{2}}\left(C_{D} \frac{A_{\max }}{h_{\max }} \sqrt{\frac{2\left(P_{p}-P_{m A}\right)}{\rho}}\right) \\
\frac{\beta}{V_{3}}\left(C_{D} \frac{A_{\max }}{h_{\max }} \sqrt{\frac{2\left(P_{p}-P_{m B}\right)}{\rho}}\right) \\
0
\end{array}\right.
$$$$
\left.\begin{array}{l} 
\\
D_{p} \\
0 \\
0 \\
0 \\
0
\end{array}\right]
$$

where $\beta$ is the damping factor, $D_{(\bullet)}$ is the displacement of the motor or pump, and $K_{(\bullet)}$ is the leakage factor of the pump or motor. Finally, $T_{\operatorname{lm} A}$ and $T_{\operatorname{lm} B}$ are a constant load torque on motors that represent the rotating shafts friction.

\section{2-D LINEARIZATION}

To analyze the system behavior or to control the original nonlinear system, a well-developed linear model can be used through linearization of the nonlinear system. This provides an approximation of the system's dynamical behavior within a neighborhood vicinity of variables and operating conditions. Hydraulic wind power systems operate under a wide range of conditions due to various disturbances such as wind speed, valve position, and load on the generators.

From (15), it can be inferred that the pressure differential variation disturbs the flow through the valve's orifices. Thus, for maintaining the flow rate, specifically through the main motor, the proportional valve's spool displacement must be adjusted to compensate for this disturbance. Intermittent wind speed imposes a variable pump speed and consequently varies the flow rate in the valve's inlet.

Even if the valve maintains the main motor's flow rate as constant, the speed can deviate from synchronous speed due to pressure variations and load torques. These types of nonlinear systems with a wide range of operating points can be linearized using multiple linear models to represent the entire system dynamics. The linearization technique used in this paper is to utilize a local linear model for each of the different plants' operating conditions.

For a wide range of operations, piecewise linearization technique can be used to cover the entire possible operation. Each model should satisfactorily describe the plant in a region around a set of specific operating points (domain). This linearized plant will have an effective range of linearization, in which the system generates a limited deviation from the original plant. The operating points out of this range result in a reduced performance as the output deviates from the nonlinear model. Hence, a new plant with shifted operating conditions is required. A 2-D or higher order inputs impose larger selection regions and consequently the linearized models may show overlap in some areas and no coverage on others. One solution might be the introduction of a new model for uncovered

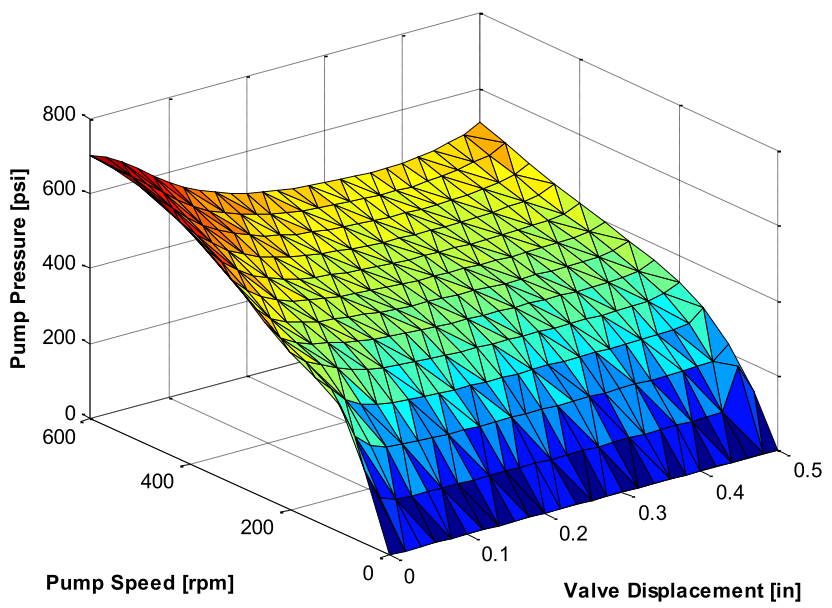

Fig. 6. Nonlinear model of hydraulic wind power transfer system for a fixed load. The valve displacement and pump pressure were varied, which resulted in pressure variations. The increase in pressure at a constant pump speed when the displacement varies is nonlinear. In addition, the pressure shows a highly nonlinear variation at a constant valve displacement and varying pump speed.

areas. This will increase the number of linearized models, and might consequently increase the overlapped areas. Hence, selection of the operating points and effective consideration of the overlapped areas in piecewise linearization becomes critical [32].

To obtain the values for the linear state space models, first, the symbolic derivate of the nonlinear state space model, mentioned in (19) and (20), around a parameterized operating point is provided. Then the selected operating points based on the described algorithm as well as the experimental system parameters are replaced into it.

\section{DISCUSSION}

As explained in Section V, a hydraulic wind power transfer system is largely sensitive to the valve position and the wind speed disturbances. The system operation due to the existence of the proportional valve is highly nonlinear. The system pressure variations due to the valve position and the wind speed are shown in Fig. 6. The surface shows a nonlinear response of the system while the load is constant. As the load changes, the system response also changes and consequently affects the rotational speed of the wind turbine. This indicates that the system requires to be linearized around multidimensional operating points. The total number of operating points can be unlimited as the surface may contain viable nodes with domains that involve a large group of operating points. Finding suitable nodes with a large domain is of interest as this optimizes the number of piecewise linearized models.

As mentioned in Section II-A, the criteria to include a possible node in a particular domain is the error and its weight, which is inversely proportional to the number of system domains claiming the node in their domain, i.e., as a particular node belongs to more domains of linearized systems, it loses its value.

In a small signal model of a linearized system, the system behavior and its linearized counterpart are similar, generating 


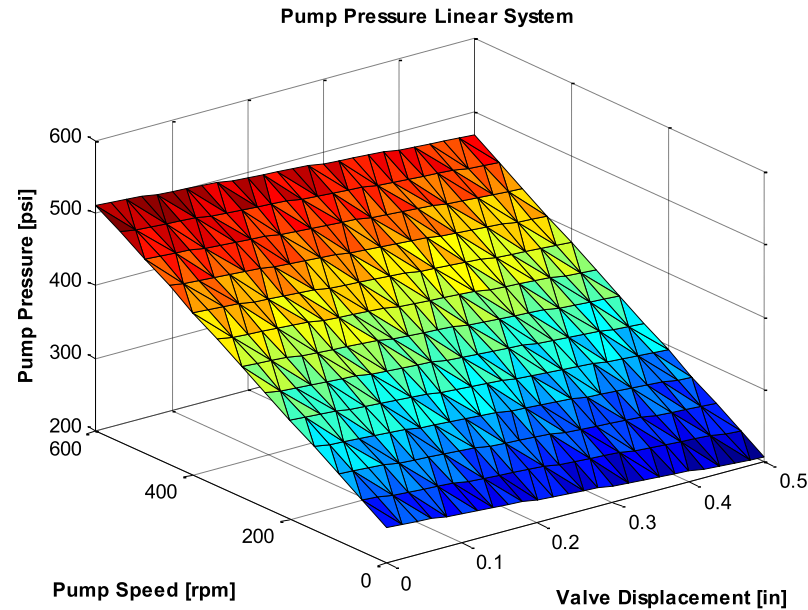

Fig. 7. Linearized model of hydraulic wind power system around all the possible operating points. The model shows that the nonlinear behavior of the pressure versus valve displacement and pump speed has been linearized.

little deviation in their response in a small enough distance from the neighboring nodes. A piecewise linearized model of the nonlinear system (Fig. 6) is shown in Fig. 7. The linearized system has many operating points that may also be influenced by disturbances in the form of measurement and system noise. In this case, the linearized model's output deviates more from the nonlinear model behavior.

This discrepancy between the linearized and the nonlinear model increases when the operating point moves further away from the linearized model effective range.

Considering an acceptable deviation range between the linearized and the nonlinear model determines the domain of each piecewise linearized model (7\% error in this paper).

A count on the number of models that share a particular node determines the weight of the node. Among all the possible nodes to be considered as operating points, an optimum number with the optimum location must be selected to minimize the number of piecewise linearized models and maximize the matching and coverage.

The range of variables in the input of a hydraulic wind power system is 200-600 rpm for shaft speed (in some systems between 10 and $30 \mathrm{rpm}$ ) and from 0 to 0.5 in for valve position. The piecewise nonlinear modeling is formulated for hydraulic wind power in three major steps as: 1) selection of optimum model number; 2) selection of operating points; and 3) adaptive combination of models to obtain model continuity. For better comparison, the introduced piecewise linear modeling is compared with an evenly distributed model selection.

\section{A. Evenly Distributed Model Selection}

For the first step, the value of the operating points was determined through evenly distributed nodes in the domains. Selecting six models, a limited error with the maximum coverage was achieved. Fig. 8 shows all the nodes that are associated to each model. Piecewise linearized models and their operating points are also shown in Fig. 8 along with the domain of the model. Overlapping and no-coverage nodes are

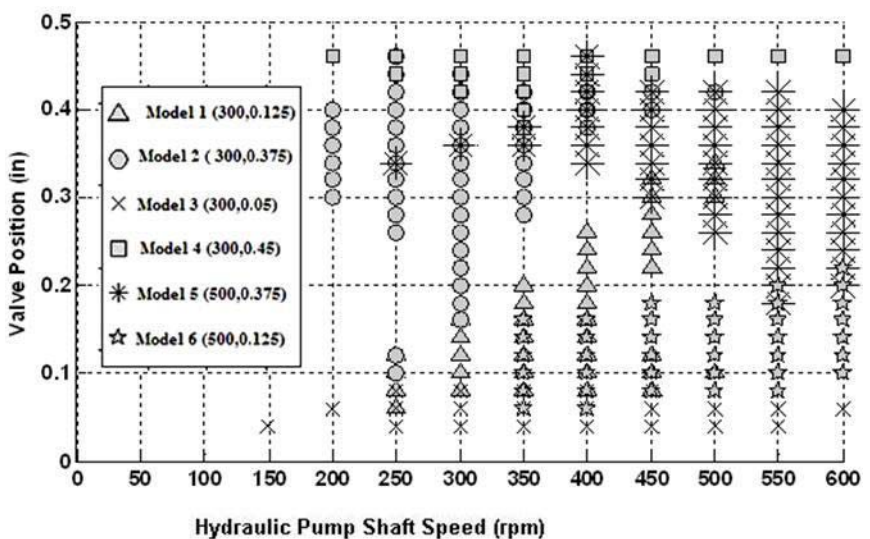

Fig. 8. Domain associated to each piecewise linearized model. A total of six models at the designated operating points were selected. Each sign shows the domain associated to the models. There are nodes that belong to no model and some nodes that belong to one or more linearized models.

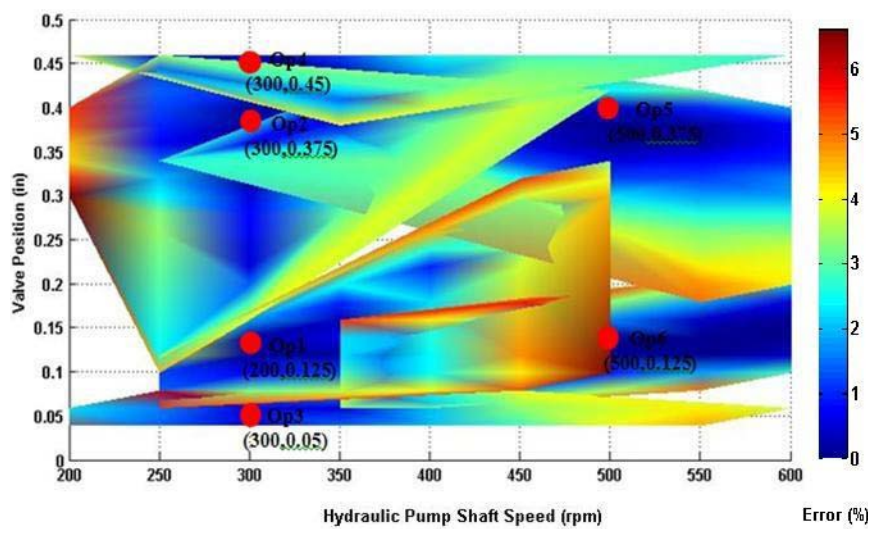

Fig. 9. Acceptable domain for a random selection of nodes and the piecewise linearized models. Colors show the error in the linearized models compared with the nonlinear model. Blue indicates low error while red-yellow indicates the high deviation in modeling. In this particular case the maximum modeling error was $7 \%$.

clearly shown with double, triple, or no signs. Fig. 9 shows a selection of the operating points and model linearization errors and their deviation from the nonlinear model. Dark areas illustrate the higher errors while the white areas demonstrate nodes out of the linearization domains and coverage.

\section{B. Adaptive Linear Continuity}

Kalman filters have been designed for the linearized models and used in multiple model adaptive mixing to determine the optimum model for overlapping and no-coverage nodes. Adaptive model-based estimation was used to estimate the output variables of hydraulic wind power as pressures of the fluid in different points of the hydraulic circuit and their comparison with that obtained from the nonlinear model. Two inputs, as proportional valve position and the hydraulic motors' shaft rotational speed, were used to determine the piecewise linearized models.

Limiting the maximum modeling error to $7 \%$, the utilization of adaptive model-based estimation improved the node coverage to $80.75 \%$ for all nodes. Fig. 10 shows the smooth coverage of the nodes. 


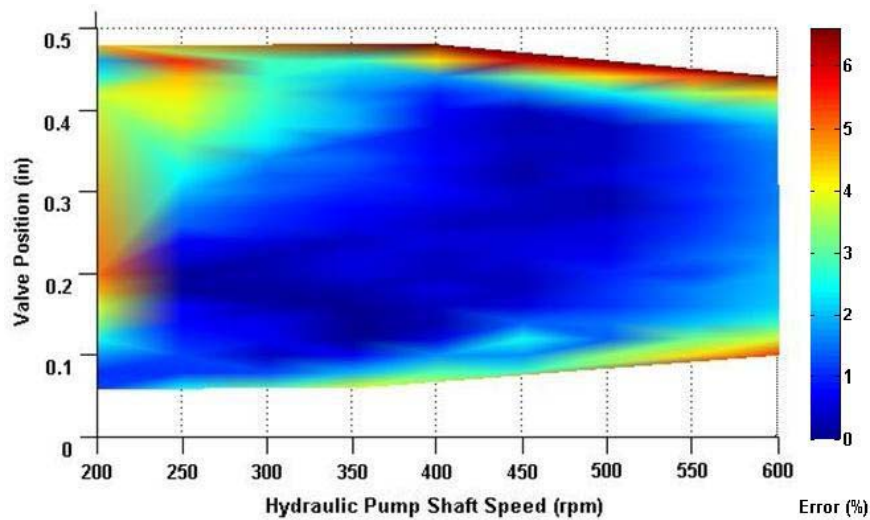

Fig. 10. Effect of adaptive model mixing on the overall model estimation and error. Red areas contain nodes with a higher error. Most of the domain nodes belong to at least one model and the entire region was converted into a low error region (blue). The areas with a high error have been shifted to the outer band of the domain where the practical operating points never reach.

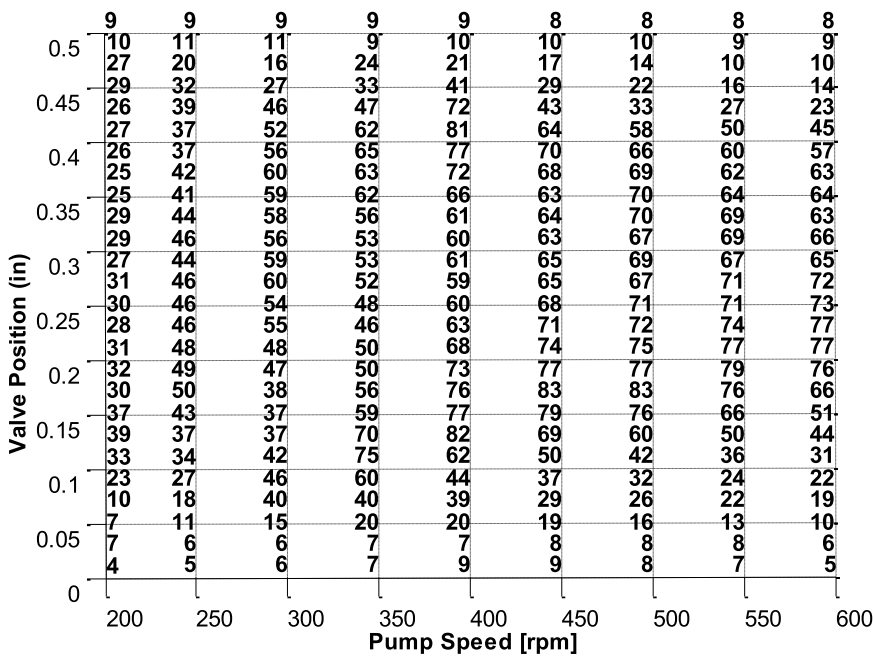

Fig. 11. Digits determine the number of models that represent a node. For instance, 20 shows that the node can possibly belong to 20 piecewise linearized models, if the modeling is continued to involve 20 models.

\section{Adaptive Model Selection}

In the first step, to select the models the values of the nodes must be calculated. The node values are the likelihood that the node belongs to the nearby model domains. The more the models cover a node, the less worthy the node becomes. Fig. 11 shows the value of each node based on the number of models that can potentially cover it. Scaling factor was used to select the optimum nodes. The gain and shape variables $\alpha$ and $\beta$ will result in various scaling factors. It is recommended to select these two variables within reasonable orders so that the scaling factor would magnify the importance of the nodes and provide a simpler representation. Through trial and error, values of 100 and 1 can be selected for $\alpha$ and $\beta$, respectively. Table II shows the selection process for $\alpha$ and $\beta$. These values result in enough resolution to select the models. The models are valued based on the weight of the nodes in their domains. The model with the highest rank covers an optimum number of nodes. Therefore, the model with the highest rank is selected as a candidate for piecewise linearized model.
TABLE II

Scaling Factor Selection. EfFect of Variables on the Scaling FACTOR. Higher SCALING FACTOR IS DESIRABle as It MAGNifies THE IMPORTANCE OF NODES IN THEIR MOdEL SELECTION

\begin{tabular}{ccc}
\hline $\begin{array}{c}\text { Scaling } \\
\text { Variables }\end{array}$ & $\begin{array}{c}\text { Model } \\
\text { Ranks }\end{array}$ & $\begin{array}{c}\text { Scaling } \\
\text { Factor }\end{array}$ \\
\hline & 1 & 0.0387 \\
\cline { 2 - 3 }$\beta=1$ & 2 & 0.0155 \\
\cline { 2 - 3 } & 3 & 0.0053 \\
\cline { 2 - 3 } & 4 & 0.0040 \\
\hline & 1 & $\mathbf{1 2 4 . 9 6 0 3}$ \\
$\boldsymbol{n y y} \boldsymbol{\beta}=\mathbf{1 0 0}$ & 2 & 73.5714 \\
\cline { 2 - 3 } & 3 & 75.8166 \\
\hline & 4 & 35.2784 \\
\hline & 1 & 3.8782 \\
$\beta=100$ & 2 & 1.5511 \\
\cline { 2 - 3 } & 3 & 0.5318 \\
\hline
\end{tabular}

TABLE III

Model Ranking in Each Iteration. Weight of Each Model IS DETERMINED AS THE NUMBER OF NODES IT INCLUDES IN ITS Domain. As The Highest Weight MODEL IS SElECTED, THE Nodes As SOCIATED TO THIS MODEL WILl BE ELIMINATED FROM THE DOMAIN

\begin{tabular}{|c|c|c|}
\hline & $\begin{array}{c}\text { Model } \\
\text { Rank }\end{array}$ & $\begin{array}{c}\text { Model } \\
\text { Weight }\end{array}$ \\
\hline \multirow{4}{*}{ Initial weigh } & 1 & 147.42 \\
\hline & 2 & 145.99 \\
\hline & 3 & 145.62 \\
\hline & 4 & 145.30 \\
\hline \multirow{4}{*}{ Iteration 1} & 1 & 145.62 \\
\hline & 2 & 130.35 \\
\hline & 3 & 130.35 \\
\hline & 4 & 124.96 \\
\hline \multirow{4}{*}{ Iteration 2} & 1 & 124.96 \\
\hline & 2 & 124.96 \\
\hline & 3 & 121.14 \\
\hline & 4 & 119.96 \\
\hline \multirow{4}{*}{ Iteration 3} & 1 & 121.14 \\
\hline & 2 & 114.66 \\
\hline & 3 & 114.66 \\
\hline & 4 & 113.46 \\
\hline \multirow{4}{*}{ Iteration 4} & 1 & 105.55 \\
\hline & 2 & 105.55 \\
\hline & 3 & 105.55 \\
\hline & 4 & 105.55 \\
\hline \multirow{4}{*}{ Iteration 5} & 1 & 97.39 \\
\hline & 2 & 94.42 \\
\hline & 3 & 94.42 \\
\hline & 4 & 91.51 \\
\hline \multirow{4}{*}{ Iteration 6} & 1 & 88.65 \\
\hline & 2 & 83.63 \\
\hline & 3 & 81.82 \\
\hline & 4 & 81.64 \\
\hline
\end{tabular}

The nodes that configure the domain of the selected models are eliminated from the weighting process. With new existing nodes, the linearization and model selection process continues.

As each model is optimized to contain the highest node weights, the model selection is inherently optimized. The model ranking and iteration process is given in Table III. As Table III shows, the first four models are ranked in each 
TABLE IV

Number of AdDED COVERED NODES IN EACH ITERATION AND THE Domain INDEX. Domain IndeX SHOWS THE NeCESSITY OF ADDING ONE MORE MODEL TO THE LIST OF LINEARIZED MODELS. ONCE THE ADDED VALUE IS LESS THAN EXPECTED, MODEL SELECTION STOPS

\begin{tabular}{cccc}
\hline Iteration & $\begin{array}{c}\text { \# of Covered } \\
\text { Nodes per Model }\end{array}$ & $\begin{array}{c}\text { Acumulative } \\
\text { \# of Nodes }\end{array}$ & $\begin{array}{c}\text { Domain Index } \\
\text { (Added/Acumulative) }\end{array}$ \\
\hline 1 & 93 & 93 & $93 / 93=1$ \\
\hline 2 & 22 & 115 & $22 / 115=0.191$ \\
\hline 3 & 17 & 132 & 0.128 \\
\hline 4 & 26 & 158 & 0.164 \\
\hline 5 & 12 & 170 & 0.070 \\
\hline 6 & 7 & 178 & $\mathbf{0 . 0 3 9}<\mathbf{0 . 0 4} \rightarrow$ Stop \\
\hline
\end{tabular}

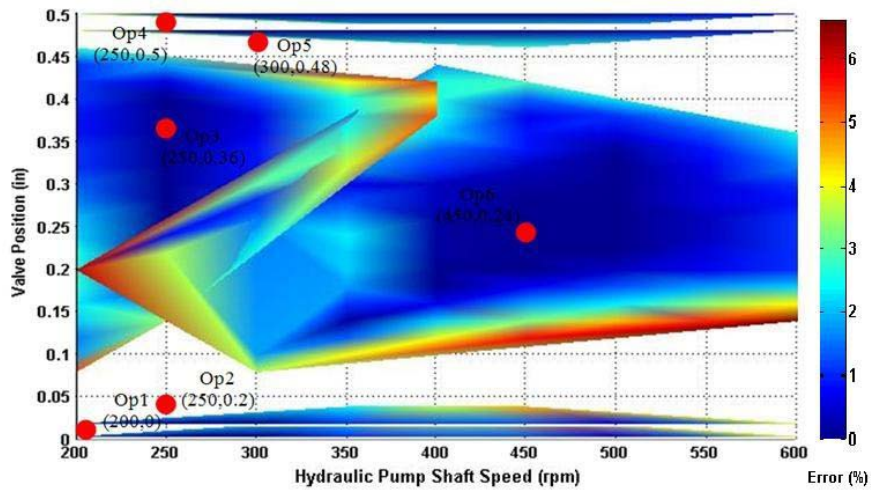

Fig. 12. Selected models through the introduced algorithm and their domains. Compared with Fig. 9, a considerable reduction in the estimation error can be observed. The target was to locate operating points and linearized models that cover most of the nodes and generate less error. See the Appendix for the linearized model parameters.

iteration and the model associated to the highest rank was selected. As the nodes were eliminated from the domains, the weight of other iterations decreased such that in the ratio of weight to number of nodes (domain index), an added model covered became a small value. At some point (selected arbitrarily), adding a model does not add enough domain coverage to the overall coverage of the system. Table IV gives the decreasing number of nodes added to the overall coverage in each iteration.

Therefore, five iterations were sufficient for this system and six models were selected to fulfill the system requirements. Table IV gives the number of added models over the total covered nodes in each iteration, i.e., the domain index.

The selected optimum piecewise linearized models are shown in Fig. 12. These models cover $86.2 \%$ of the entire domain and generate errors less than $7 \%$. The performance of adaptive model mixing to optimize the coverage and minimize the overall error is shown in Fig. 13.

As can be seen by the selected models from the introduced algorithm, the overall accuracy of the adaptive piecewise linearization has increased by $5.45 \%$. In addition, comparing Figs. 10 and 13, it can be concluded that besides the increase in the overall coverage of the node domains, the error between the nonlinear system and the multiple model adaptive

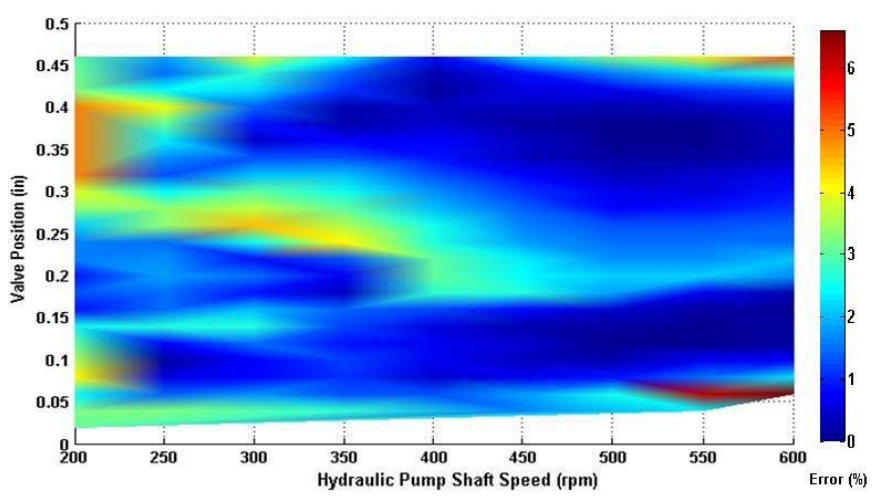

Fig. 13. Adaptive model mixing increased the entire area coverage and reduced the overall error in modeling the noncovered nodes. The outer band of operating points has less error than what is shown in Fig. 10. This shows the improvement that the introduced algorithm can provide along with the adaptive model mixing.

estimation was optimized. These improvements are observed in the accurate increase of the state variable estimation as an important measure in approximate piecewise linearization.

\section{CONCLUSION}

This paper introduced an automatic algorithm to select the optimum operating points for piecewise linearization of nonlinear systems with multidimensional inputs. The algorithm is operated based on the weighting factor for each node to be included in the domain of all the possible models. All the possible models are ranked and the model with the highest weight was selected. The domain and model were removed from the ranking and model selection was continued until reaching a point where adding one more model to the set of linearized models did not involve a significant number of nodes. At this point, there were nodes that might belong to one, many, or none of the models. An adaptive mixing was introduced that allocated a probability to each model for each operating point to keep the domain continuity and minimum model linearization error. Without loss of generality, the introduced algorithm was formulated for a 2-D input wind power system. The introduced systematic linearization approach was compared with a random selection of models. The coverage of domains and the overall modeling error were significantly improved.

\section{ACKNOWLEDGMENT}

The authors would like to thank the anonymous reviewers for their suggestions and comments.

\section{REFERENCES}

[1] X. Liu and Y. Yuan, "Nonlinear system modeling and application based on system equilibrium manifold and expansion model," J. Comput. Nonlinear Dyn., vol. 9, no. 2, p. 021013, 2014.

[2] A. Isidori, Nonlinear Control Systems. Springer, 1995.

[3] J.-J. E. Slotine and J. K. Hedrick, "Robust input-output feedback linearization," Int. J. Control, vol. 57, no. 5, pp. 1133-1139, 1993.

[4] K. Park, H. Chung, and J. G. Lee, "Point stabilization of mobile robots via state-space exact feedback linearization," Robot. Comput.-Integr. Manuf., vol. 16, no. 5, pp. 353-363, 2000.

[5] F. Belkhouche, "Trajectory-based optimal linearization for nonlinear autonomous vector fields," IEEE Trans. Circuits Syst. I, Reg. Papers, vol. 52, no. 1, pp. 127-138, Jan. 2005. 
[6] Q. Gan and C. J. Harris, "Fuzzy local linearization and local basis function expansion in nonlinear system modeling," IEEE Trans. Syst., Man, Cybern. B, Cybern., vol. 29, no. 4, pp. 559-565, Aug. 1999.

[7] W. Kang, "Approximate linearization of nonlinear control systems," in Proc. 32nd IEEE Conf. Decision Control, Dec. 1993, pp. 2766-2771.

[8] L. Socha, Linearization Methods for Stochastic Dynamic Systems, vol. 730. Springer, 2008.

[9] Y. Zhang, S. Sankaranarayanan, and F. Somenzi, "Piecewise linear modeling of nonlinear devices for formal verification of analog circuits," in Proc. Formal Methods Comput.-Aided Design (FMCAD), 2012, pp. 196-203.

[10] H. Amin, K. M. Curtis, and B. R. Hayes-Gill, "Piecewise linear approximation applied to nonlinear function of a neural network," IEE Proc.Circuits, Devices Syst., vol. 144, no. 6, pp. 313-317, Dec. 1997.

[11] K. Holmberg, "Solving the staircase cost facility location problem with decomposition and piecewise linearization," Eur. J. Oper. Res., vol. 75 , no. 1 , pp. 41-61, 1994.

[12] S. Oweis and S. Omekanda, "A graphic user interface for 3D segmentation of nonlinear functions," Int. J. Adv. Res. Comput. Commun. Eng., vol. 3, no. 2, pp. 5762-5766, 2014.

[13] G. K. Lowe and M. A. Zohdy, "Modeling nonlinear systems using multiple piecewise linear equations," Nonlinear Anal., Model. Control, vol. 15, no. 4, pp. 451-458, 2010.

[14] Y. Tarte and Y. Chen, "Wiener system identification with four-segment and analytically invertible nonlinearity model," in Proc. Amer. Control Conf. (ACC), 2007, pp. 4339-4344.

[15] S. Ghosh, A. Ray, D. Yadav, and B. M. Karan, "A genetic algorithm based clustering approach for piecewise linearization of nonlinear functions," in Proc. Int. Conf. Devices Commun. (ICDeCom), 2011, pp. 1-4.

[16] P. P. Mavridis and G. V. Moustakides, "Simplified Newton-type adaptive estimation algorithms," IEEE Trans. Signal Process., vol. 44, no. 8, pp. 1932-1940, Aug. 1996

[17] G.-O. Glentis, K. Berberidis, and S. Theodoridis, "Efficient least squares adaptive algorithms for FIR transversal filtering," IEEE Signal Process. Mag., vol. 16, no. 4, pp. 13-41, Jul. 1999.

[18] A. Izadian, P. Khayyer, and P. Famouri, "Fault diagnosis of time-varying parameter systems with application in MEMS LCRs," IEEE Trans. Ind. Electron., vol. 56, no. 4, pp. 973-978, Apr. 2009.

[19] H. Stearns and M. Tomizuka, "Multiple model adaptive estimation of satellite attitude using MEMS gyros," in Proc. Amer. Control Conf. (ACC), 2011, pp. 3490-3495.

[20] M. Vaezi and A. Izadian, "Multiple-model adaptive estimation of a hydraulic wind power system," in Proc. 39th Annu. Conf. IEEE Ind. Electron. Soc. (IECON), Nov. 2013, pp. 2111-2116.

[21] P. D. Hanlon and P. S. Maybeck, "Multiple-model adaptive estimation using a residual correlation Kalman filter bank," IEEE Trans. Aerosp. Electron. Syst., vol. 36, no. 2, pp. 393-406, Apr. 2000.

[22] J. Valappil and C. Georgakis, "Systematic estimation of state noise statistics for extended Kalman filters," AIChE J., vol. 46, no. 2, pp. 292-308, 2000.

[23] P. Eide and P. Maybeck, "An MMAE failure detection system for the F-16," IEEE Trans. Aerosp. Electron. Syst., vol. 32, no. 3, pp. 1125-1136, Jul. 1996.

[24] A. Izadian, N. Girrens, and P. Khayyer, "Renewable energy policies: A brief review of the latest U.S. and E.U. policies," IEEE Ind. Electron. Mag., vol. 7, no. 3, pp. 21-34, Sep. 2013.

[25] S. Eriksson, H. Bernhoff, and M. Leijon, "Evaluation of different turbine concepts for wind power," Renew. Sustain. Energy Rev., vol. 12, no. 5, pp. 1419-1434, 2008.

[26] M. Deldar, A. Izadian, M. Vaezi, and S. Anwar, "Modeling of a hydraulic wind power transfer utilizing a proportional valve," IEEE Trans. Ind. Appl., vol. 51, no. 2, pp. 1837-1844, Mar./Apr. 2015.

[27] M. Vaezi and A. Izadian, "Piecewise affine system identification of a hydraulic wind power transfer system," IEEE Trans. Control Syst. Technol., vol. 23, no. 6, pp. 2077-2086, Nov. 2015.

[28] A. Izadian, "Central wind turbine power generation awarded," U.S Patent 8878384, Nov. 04, 2014.

[29] J.-Z. Bai, A.-G. Xie, X.-H. Yu, and L.-K. Zhou, "Simulation model of hydraulic turbine speed control system and its parameters identification based on resilient adaptive particle swarm optimization algorithm," in Proc. Asia-Pacific Power Energy Eng. Conf. (APPEEC), 2010, pp. 1-4.

[30] M. Vaezi and A. Izadian, "Control of a hydraulic wind power transfer system under disturbances," in Proc. Int. Conf. Renew. Energy Res. Appl. (ICRERA), 2014, pp. 886-890.

[31] H. E. Merritt, Hydraulic Control Systems. New York, NY, USA: Wiley, 1967.

[32] M. Vaezi, A. Izadian, and M. Deldar, "A model linearization technique for hydraulic wind power systems," in Proc. Power Energy Conf. Illinois (PECI), 2014, pp. 1-5.

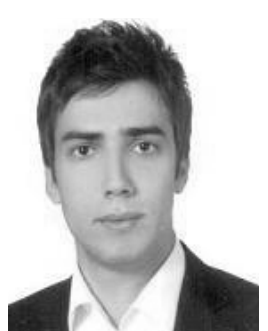

automotive systems.
Masoud Vaezi received the B.Sc. degree in mechanical engineering from the $\mathrm{K}$. N. Toosi University of Technology, Tehran, Iran, in 2012, and the M.Sc. degree in mechanical engineering from The Purdue School of Engineering and Technology, Indianapolis, IN, USA, in 2014.

$\mathrm{He}$ was a Research Assistant with the Energy Systems and Power Electronics Laboratory. His current research interests include modeling, simulation, and control of integrated systems, including robotic systems, power systems, and

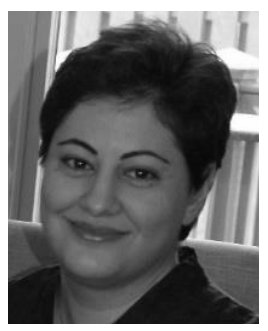

Pardis Khayyer (S'06-M'15) received the M.S. degree in electrical engineering from West Virginia University, Morgantown, WV, USA, in 2008, and the Ph.D. degree in electrical engineering from The Ohio State University, Columbus, OH, USA, in 2013.

She was with the Department of Electrical and Computer Engineering and the Center for Automotive Research, The Ohio State University. She has six years of industry experience as a Controls and Diagnostics Engineer. Her current research interests include application of control theory in automotive systems and power systems.

Dr. Khayyer is a member of Eta Kappa Nu, Tau Beta Pi, Phi Kappa Phi, and Sigma Xi Honor Societies.

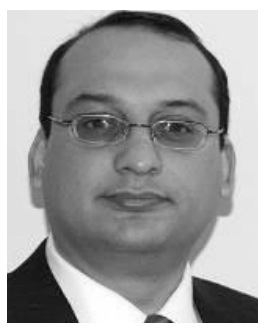

Afshin Izadian (S'04-M'09-SM'10) received the M.S. degree in electrical engineering from the Iran University of Science and Technology, Tehran, Iran, in 2002, and the Ph.D. degree in electrical engineering from West Virginia University, Morgantown, WV, USA, in 2008.

$\mathrm{He}$ was a Post-Doctoral Researcher with the University of California at Los Angeles, Los Angeles, CA, USA, in 2009. He is currently an Associate Professor with The Purdue School of Engineering and Technology, Indianapolis, IN, USA. He is the Founding Director of the Energy Systems and Power Electronics Laboratory, IUPUI. His current research interests include application of control systems in renewable energy, energy conversion, power electronics, and electric power systems.

Dr. Izadian is a Lifetime member of Etta Kappa $\mathrm{Nu}$, Tau Beta Pi, and Sigma Xi. 